Pesq. Vet. Bras. 37(8):797-801, agosto 2017 DOI: $10.1590 / \mathrm{S} 0100-736 \mathrm{X} 2017000800003$

\title{
Doenças parasitárias em bovinos e ovinos no sul do Brasil: frequência e estimativa de perdas econômicas ${ }^{1}$
}

\author{
Plínio Aguiar de Oliveira² ${ }^{2}$ Jerônimo Lopes Ruas ${ }^{3}$, Franklin Riet-Correa ${ }^{4}$, \\ Ana Carolina B. Coelho ${ }^{5}$, Bianca Lemos Santos ${ }^{5}$, Clairton Marcolongo-Pereira ${ }^{6}$, \\ Eliza Simone Viegas Sallis ${ }^{7}$ e Ana Lucia Schild ${ }^{3 *}$
}

\begin{abstract}
Oliveira P.A., Ruas J.L., Riet-Correa F., Coelho A.C.B., Santos B.L., Marcolongo-Pereira C., Sallis E.S.V. \& Schild A.L. 2017. [Parasitic diseases of cattle and sheep in southern Brazil: frequency and economic losses estimate.] Doenças parasitárias em bovinos e ovinos no sul do Brasil: frequência e estimativa de perdas econômicas. Pesquisa Veterinária Brasileira 37(8):797-801. Departamento de Parasitologia e Microbiologia, Instituto de Biologia, Universidade Federal de Pelotas, Campus Universitário s/n, Pelotas, RS 96010-900, Brazil. E-mail: alschild@terra.com.br

A survey of parasitic diseases of cattle and sheep diagnosed in Southern Brazil, from January 1978 to December 2014, was conducted in the Laboratório Regional de Diagnóstico, of Universidade Federal de Pelotas. In cattle $10.1 \%$ of all cases diagnosed were parasitic diseases, of which the most common was tick fever with $55.1 \%$ of cases. Mixed gastrintestinal parasitosis was diagnosed in $22.35 \%$ of cases, hemonchosis in $4.36 \%$, dyctiocaulosis in $3.52 \%$, fluke infection in $2.68 \%$, and eimeriosis in $1.84 \%$ of the cases. In sheep $33.6 \%$ of the diagnoses was parasitosis. In sheep mixed gastrintestinal parasitosis was the most frequent with $42.7 \%$ of the cases, hemonchosis was observed in $35.4 \%$, coenurosis in $9.1 \%$ and fluke infection in $4.4 \%$ of the cases. It is estimated that losses through mortality resulting from parasitic diseases in cattle is approximately $\mathrm{R} \$ 16.968 .000 /$ year. In sheep the economic losses through mortality caused by parasites is about $\mathrm{R} \$ 2.016 .000 /$ year. The results of this survey realize the importance of parasitic diseases in ruminants in Southern Brazil, based on data from more than three decades of diagnostic. It was concluded that, in cattle in southern Rio Grande do Sul, the TPB agents are the main cause of economic losses, followed by mixed parasitic infections. In sheep mixed gastrointestinal parasitosis and hemoncose together represent more than $70 \%$ of the diagnoses performed.
\end{abstract}

INDEX TERMS: Parasitic diseases, cattle, sheep.

\footnotetext{
${ }^{1}$ Recebido em 6 de agosto de 2015.

Aceito para publicação em 13 de setembro de 2016.

${ }^{2}$ Curso de Pós-Graduação em Parasitologia, Instituto de Biologia, Universidade Federal de Pelotas (UFPel), Campus Universitário Capão do Leão s/n, Capão do Leão, RS 96010-900, Brasil.

${ }^{3}$ Laboratório Regional de Diagnóstico, UFPel, Campus Universitário Capão do Leão s/n, Capão do Leão, RS 96010-900, Brasil. *Autor para correspondência: alschild@terra.com.br

${ }^{4}$ Hospital Veterinário, Centro de Saúde e Tecnologia Rural (CSTR), Universidade Federal de Campina Grande (UFCG), Campus de Patos, Avenida Universitária s/n, Santa Cecília, Patos, PB 58708-110, Brasil.

${ }^{5}$ Curso de Pós-Graduação em Veterinária, Faculdade de Veterinária, UFPel, Campus Universitário Capão do Leão s/n, Capão do Leão, RS 96010-900, Brasil.

${ }^{6}$ Faculdade de Veterinária, Centro Universitário Ritter dos Reis (UniRitter), Rua Orfanotrofio 555, Alto Teresópolis, Porto Alegre, RS 90840-440, Brasil.

${ }^{7}$ Departamento de Patologia, Faculdade de Veterinária, UFPel, Campus Universitário Capão do Leão s/n, Capão do Leão, RS 96010-900, Brasil.
}

RESUMO.- Foi realizado um levantamento das doenças parasitárias de bovinos e ovinos diagnosticadas na região sul do Rio Grande do Sul, de janeiro de 1978 a dezembro de 2014 no Laboratório Regional de Diagnóstico da Universidade Federal de Pelotas. Em bovinos $10,1 \%$ de todos os diagnosticos tratavam-se de parasitoses, das quais a mais frequente foi a tristeza parasitária bovina, com 55,1\% dos surtos. As parasitoses gastrintestinais mistas foram diagnosticadas em $22,35 \%$ dos casos, a hemoncose em $4,36 \%$, a dictiocaulose em 3,52\%, a fasciolose em 2,68\% e a eimeriose em 1,84\%. Em ovinos 33,6\% dos diagnósticos realizados eram parasitoses, sendo as mais frequentes as parasitoses gastrintestinais mistas $(42,7 \%)$, a hemoncose $(35,4 \%)$, a coenurose $(9,1 \%)$ e a fasciolose $(4,4 \%)$. Estima-se que as perdas somente por mortalidade, decorrentes de doenças parasitárias em bovinos somam aproximadamen- 
te $\mathrm{R} \$ 16.968 .000 /$ ano. Na espécie ovina as perdas econômicas causadas por mortalidade de origem parasitária são de aproximadamente $\mathrm{R} \$ 2.016 .000 /$ ano na região sul do Rio Grande do Sul. Foi possível concluir que, em bovinos, na região sul do Rio Grande do Sul, os agentes da TPB são os principais causadores de perdas econômicas, seguidos pelas parasitoses mistas. Em ovinos a parasitose gastrintestinal mista e a hemoncose, juntas, representam mais de $70 \%$ dos diagnósticos realizados.

TERMOS DE INDEXAÇÃO: Parasitoses, bovinos, ovinos.

\section{INTRODUÇÃO}

Em levantamento de dados do Laboratório Regional de Diagnóstico da Faculdade de Veterinária da Universidade Federal de Pelotas (LRD/UFPel) tem sido observado que na região sul do Rio Grande do Sul aproximadamente 18,1\% e $66,8 \%$ dos diagnósticos em bovinos e ovinos, respectivamente, referem-se a doenças parasitárias (Schild et al. 2015). Isso demonstra a importância dessas enfermidades como causa de prejuízos econômicos para a pecuária da região. 0 diagnóstico etiológico é fundamental na formação de estratégias de controle das doenças parasitárias (Ruas \& Berne 2007, Borges et al. 2013).

A área de influência do LRD/UFPel compreende 22 municípios da região Sul do Rio Grande do Sul e possui uma população aproximada de 2.800 .000 bovinos e 1.000 .000 de ovinos (IBGE 2012) em uma área aproximada de 44.000 $\mathrm{km}^{2}$. Neste contexto, as doenças parasitárias são responsáveis por elevadas perdas econômicas, devido à morbidade e a mortalidade, com redução da produção dos animais, dificultando a manutenção e melhoramento dos rebanhos (Perry \& Randolph 1999, Amarante 2004).

As parasitoses são uma das principais causas da redução de produtividade dos ruminantes. Geralmente, não ocorrem com a presença de um único gênero parasitário, mas sim de forma mista. A ocorrência dos parasitos depende, por exemplo, de elementos como: temperatura, precipitação pluviométrica, solo, topografia, tipo e manejo da pastagem, espécie, raça, idade, estado fisiológico e nutricional e manejo dos animais (Molento 2005, Ruas \& Berne 2007). 0 conhecimento das doenças parasitárias, bem como suas particularidades regionais é importante para a realização de um programa de controle eficiente (Costa et al. 2009). Esse deve ser sustentado na presença, distribuição, dinâmica populacional, incidência de gêneros e espécies potencialmente patogênicas na região estudada (Ruas \& Berne 2007).

Os objetivos do presente estudo foram identificar e avaliar a partir da análise de protocolos de necropsia do LRD/ UFPel, as principais doenças parasitárias que ocorreram na região Sul do Rio Grande do Sul em ruminantes, entre janeiro de 1978 e dezembro de 2014, estabelecendo os aspectos epidemiológicos mais comuns, bem como estimar as perdas econômicas delas decorrentes.

\section{MATERIAL E MÉTODOS}

Para o estudo foram pesquisados nos arquivos LRD/UFPel os protocolos de necropsias realizadas no laboratório ou a campo e protocolos de materiais remetidos por veterinários particulares, entre janeiro de 1978 e dezembro de 2014. Foram identificados os protocolos de enfermidades parasitárias em ruminantes (bovinos e ovinos) resgatando-se dados epidemiológicos referentes à procedência dos animais, época do ano, tipo de criação, idade, sexo, raça e aptidão. Foram considerados todos os protocolos cujos diagnósticos haviam sido confirmados pelos sinais clínicos, necropsias realizadas e/ou exames complementares tais como o coproparasitológico, esfregaço sanguíneo, impressão de órgãos e raspado de pele. Os diagnósticos realizados foram agrupados em diferentes categorias de acordo com a natureza do agente etiológico. 0 diagnóstico de parasitose gastrintestinal mista em bovinos e ovinos foi considerado quando mais de um parasito era identificado no exame realizado, sem o predomínio de um, sendo atribuído um diagnóstico específico quando existia o predomínio de um parasito.

Para a estimativa de perdas econômicas considerou-se a mortalidade anual de bovinos e ovinos de 5\% (Riet-Correa \& Medeiros 2001, SEAPA 2010) em um rebanho aproximado de 2.800 .000 bovinos e 1.000.000 de ovinos (IBGE 2012), considerando-se o preço médio de um bovino de $\mathrm{R} \$ 1.200,00$ e de um ovino de $\mathrm{R} \$ 120,00$.

\section{RESULTADOS}

No período de janeiro de 1978 a dezembro de 2014 foram recebidos no LRD/ UFPel para diagnóstico 5.887 materiais de bovinos, dos quais, 10,1\% (595) tratavam-se de parasitoses; e 816 materiais de ovinos, dos quais 33,6\% (274) eram referentes a parasitoses. 0 material analisado foi 32,1\% (191/595) de cadáveres bovinos, 46,3\% (187/595) órgãos, 35,1\% (142/595) amostras de fezes, $15,6 \%(63 / 595)$ amostras de sangue e $0,5 \%(2 / 595)$ raspados de pele. Na espécie ovina foram analisados em $39 \%$ (107/274) cadáveres, 77,8 \% (130/274) amostras de fezes, $19,8 \%$ (33/274) órgãos e 2,4\% (4/274) raspados de pele.

Quanto à raça dos bovinos, 42,8\% (254/595) eram de raças europeias (Hereford, Angus, Devon, Shorthorn, Holandesa, Jersey), 10,1\% (60/595) de raças continentais (Charolês, Normanda, Limousin, Lincoln Red), 5,9\% (35/595) zebuínas (Nelore, Brahma, Gir, Tabapuã) e 21,8\% (130/595) cruzas. Em 19,5\% (116/595) dos protocolos a raça não foi informada. Em relação à aptidão dos bovinos, $20,3 \%(121 / 595)$ eram de raças leiteiras criados em regime de semi-confinamento e 79,6\% (474/595) eram bovinos de corte criados extensivamente.

Em 328 surtos dos 595 protocolos de bovinos analisados, o diagnóstico era de tristeza parasitária bovina (TPB). Destes, 45\% (135/328) tratava-se de babesiose por $B a-$ besia bovis, 34\% (101/328) anaplasmose, 4,7\% (14/328) eram infecções mistas e oito 2,7\% (8/328) eram babesiose por Babesia bigemina. Em 13,1\% (39/328) dos protocolos o diagnóstico constava como babesiose por Babesia spp.; em 9,45\% (31) o diagnóstico era de TPB sem a identificação do agente.

No Quadro 1 são apresentados os percentuais das parasitoses observadas em bovinos no período do estudo. A idade dos bovinos afetados pelas principais parasitoses, a época de ocorrência e os índices de morbidade, mortalidade e letalidade das principais parasitoses em bovinos são apresentados no Quadro 2.

0 número de surtos de parasitose gastrintestinal mista em bovinos representou $22,35 \%$ das parasitoses, sendo os principais agentes os gêneros Trichostrongylus, Cooperia, Ostertagia, Strongyloides, Nematodirus e Oesophagostomum. 
Quanto à raça e aptidão dos ovinos, 22,62\% (62/274) eram de raças carniceiras (Texel, Suffolk, Ile de France, Hampshire Down), 6,56\% (18/274) de raças laneiras (Merino, Ideal), 24,08\% (66/274) raças de dupla aptidão (Corriedale, Romney Marsh) e 35,4\% (97/274) cruzas. Em 11,31\% (31/274) protocolos a raça não foi informada.

As parasitoses diagnosticadas em ovinos são apresentadas no Quadro 1. As parasitoses gastrintestinais foram diagnosticadas em 117 oportunidades representando $42,7 \%$ dos surtos analisados nos protocolos do LRD/ UFPel. Os dados epidemiológicos, a morbidade, mortalidade e letalidade das principais parasitoses diagnosticadas são apresentados no Quadro 3. No caso das parasitoses gastrintestinais mistas os principais gêneros encontrados foram Cooperia, Ostertagia, Strongyloides, Trichostrongylus e Haemonchus.

\section{Quadro 1. Diagnósticos de parasitose em bovinos e ovinos realizados na região Sul do Rio Grande do Sul, entre 1 978 e 2014}

\begin{tabular}{|c|c|c|c|}
\hline Parasitoses & $\begin{array}{c}\text { Bovinos } \\
\text { № de surtos }(\%)\end{array}$ & Parasitoses & $\begin{array}{c}\text { Ovinos } \\
\text { № surtos (\%) }\end{array}$ \\
\hline $\mathrm{TPB}^{\mathrm{a}}$ & $328(55,12 \%)$ & $\mathrm{PGM}^{\mathrm{b}}$ & $117(42,7 \%)$ \\
\hline PGM2 & $133(22,35 \%)$ & Hemoncose & $97(35,4 \%)$ \\
\hline Hemoncose & $26(4,36 \%)$ & Coenurose & $25(9,1 \%)$ \\
\hline Dictiocaulose & $21(3,52 \%)$ & Fasciolose & $12(4,4 \%)$ \\
\hline Fasciolose & $16(2,68 \%)$ & Equinococose & $5(1,8 \%)$ \\
\hline Eimeriose & $11(1,84 \%)$ & Sarna psoróptica & $4(1,5 \%)$ \\
\hline Neosporose $e^{c}$ & $9(1,51 \%)$ & Oestrose & $3(1,1 \%)$ \\
\hline Tricostrongilose & $6(1 \%)$ & Ostertagiose & $3(1,1 \%)$ \\
\hline Equinococose & $6(1 \%)$ & Trichostrongilose. & $2(0,7 \%)$ \\
\hline Ostertagiose & $6(1 \%)$ & Cisticercose & $2(0,7 \%)$ \\
\hline Granuloma parasitário & $6(1 \%)$ & Enterite parasitária & a $2(0,7 \%)$ \\
\hline Paramfistomose & $5(0,84 \%)$ & Dictiocaulose & $1(0,4 \%)$ \\
\hline Enterite parasitária $^{\mathrm{d}}$ & $5(0,84 \%)$ & Paramfistomose & $1(0,4 \%)$ \\
\hline Coenurose & $3(0,5 \%)$ & TOTAL & 274 \\
\hline Cisticercose & $3(0,5 \%)$ & & \\
\hline Monieziose & $3(0,5 \%)$ & & \\
\hline Oncocercose & $2(0,33 \%)$ & & \\
\hline Toxoplasmose & $2(0,33 \%)$ & & \\
\hline Sarna psoróptica & $1(0,16 \%)$ & & \\
\hline Piolho (Damalinia bovis) & $1(0,16 \%)$ & & \\
\hline Cooperiose & $1(0,16 \%)$ & & \\
\hline Criptosporidiose & $1(0,16 \%)$ & & \\
\hline TOTAL & 595 & & \\
\hline
\end{tabular}

a $\mathrm{TPB}=$ tristeza parasitária bovina, ${ }^{\mathrm{b}} \mathrm{PGM}=$ parasitose gastrintestinal mista, ${ }^{\mathrm{c}}$ Refere-se a surtos de abortos em bovinos de leite, ${ }^{\mathrm{d}}$ Refere-se diagnósticos morfológicos de órgãos enviados em formol.

\section{DISCUSSÃO}

Os resultados observados neste trabalho dão conta da importância das enfermidades parasitárias em bovinos e ovinos na região Sul do Rio Grande do Sul. O presente estudo demonstrou que a TPB é a parasitose mais frequente na região de influência do LRD, representando $55,1 \%$ dos casos de doenças parasitárias diagnosticadas em bovinos. Resultado semelhante foi observado no semiárido brasileiro, onde a TPB foi a principal doença parasitária diagnosticada em bovinos (Costa et al. 2009).

As parasitoses gastrintestinais mistas em bovinos representaram $22,5 \%$ de todas as doenças causadas por parasitos. Em um levantamento realizado entre 2000 e 2007 na Paraíba, observou-se um percentual de 0,09\% de parasitoses diagnosticadas em relação ao total de casos diagnosticados na espécie bovina, a baixa frequência de parasitismo gastrintestinal pode ser devido às características da criação na região, caracterizada por baixa lotação e desmame dos animais com um ano ou mais, além das condições climáticas desfavoráveis a sobrevivência do parasito (Costa et al. 2009). Deve destacar-se que as doenças parasitárias estão entre as mais importantes no mundo como causa de prejuízos econômicos para a bovinocultura (Nari \& Rizzo 2004). No presente trabalho observou-se que as parasitoses mistas ocorreram durante todo o ano sem uma predominância estacional, conforme observações anteriores que mencionam que a infecção por nematódeos gastrintestinais em bovinos no Rio Grande do Sul ocorre o ano todo variando a intensidade de acordo com as condições climáticas (Ruas \& Berne 2007).

A hemoncose nos bovinos representou $4,4 \%$ dos diagnósticos das parasitoses. Em bovinos a campo, sem sinais clínicos, as contagens de Haemonchus spp. são geralmente baixas, demonstrando um equilíbrio entre o hospedeiro e o parasito. Fatores como manejo, confinamento, alimentação e introdução de animais de outras regiões podem alterar esse equilíbrio em favor do parasito (Nari \& Rizzo 2004) e isso, aparentemente, influiu na ocorrência dos surtos da parasitose no período do estudo.

Este estudo demonstrou que outras parasitoses como dictiocaulose e eimeriose ocorrem com uma baixa frequência na região estudada. No entanto, deve ser considerado que a eimeriose é uma parasitose que ocorre principalmente em bezerros confinados (Almeida et al. 2006) e a maioria dos casos descritos neste trabalho $(79,6 \%)$ se refere a bovi-

Quadro 2. Faixa etária, época de ocorrência, morbidade, mortalidade e letalidade das principais doenças parasitárias em bovinos diagnosticadas na região Sul do Rio Grande do Sul, no período de 1978-2014

\begin{tabular}{lcccccc}
\hline Diagnóstico & FE $^{\mathrm{a}}$ meses (média) & Época & Animais sob risco & Morb $^{\mathrm{c}}(\%)$ & Mort (\%) & Let(\%) \\
\hline TPBd4 & $2-112(26,5)$ & Dez a Jun & 61346 & 3 & 1,3 & 44 \\
PGM $^{\mathrm{e}}$ & $1-51(14)$ & Todo ano & 7395 & 17,5 & 4,6 & 26,4 \\
Hemoncose & $4-20(12,5)$ & Mar a Jun & 1197 & 22,8 & 3,7 & 16,1 \\
Dictiocaulose & $3-36(12)$ & Jun a Ago & 604 & 19,5 & 3,8 & 84,4 \\
Fasciolose & $18-96(40)$ & Mar e Abr, Nov e Dez & 2285 & 6,5 & 4,7 & 77,5 \\
Eimeriose & $3-48(17)$ & Jan a Abr & 418 & 20 & 0,87 & 4,8
\end{tabular}

${ }^{\mathrm{a}} \mathrm{FE}$ = faixa etária, ${ }^{\mathrm{b}}$ Foram somados todos os bovinos de cada surto para o cálculo de morbidade, mortalidade e letalidade ${ }^{\mathrm{c}}$ Morb $=$ morbidade, Mort = mortalidade, Let = letalidade, $\mathrm{d}$ TPB = tristeza parasitária bovina, e PGM = parasitose gastrintestinal mista. 


\begin{tabular}{|c|c|c|c|c|c|c|}
\hline Diagnóstico & $\begin{array}{l}\text { FE }^{a} \text { meses } \\
\text { (média) }\end{array}$ & Época & $\begin{array}{l}\text { Animais } \\
\text { sob risco }^{\text {b }}\end{array}$ & $\operatorname{Morb}^{c}(\%)$ & Mort (\%) & Let (\%) \\
\hline $\mathrm{PGM}^{\mathrm{d}}$ & $1-48(24,6)$ & Out a Jan & 3425 & 15,1 & 8 & 52,7 \\
\hline Hemoncose & $5-108(33,8)$ & Jan a Abr & 3937 & 5,3 & 3,7 & 70,1 \\
\hline Coenurose & $11-72(22,8)$ & Dez a Mar & 9790 & 1,8 & 1,4 & 76,1 \\
\hline Fasciolose & $12-24(16,5)$ & Abr & 2123 & 6,4 & 3,6 & 55,9 \\
\hline
\end{tabular}

${ }^{\mathrm{a}} \mathrm{FE}=$ faixa etária, ${ }^{\mathrm{b}}$ Foram somados todos os ovinos de cada surto para o cálculo de morbidade, mortalidade e letalidade, ${ }^{\mathrm{c}}$ Morb $=$ morbidade, Mort $=$ mortalidade, Let $=$ letalidade, ${ }^{\mathrm{d}} \mathrm{PGM}=$ parasitose gastrintestinal mista.

nos criados a campo. Com relação à dictiocaulose, é provável que o uso de anti-helmínticos empregados no controle da parasitose gastrintestinal tenha influenciado o número de casos de parasitose pulmonar, visto que a maioria das drogas anti-helmínticas é eficaz contra Dictyocaulus spp. (Ruas 2007, Fiel et al. 2011). Além disso, nessas parasitoses, a ocorrência de resistência não tem sido mencionada na região.

Por outro lado, a fasciolose é uma parasitose endêmica na região Sul do Rio Grande do Sul e um alto percentual de condenação de fígados tem sido mencionado em diversos trabalhos variando de $18,6 \%$ a $19,6 \%$ (Cunha et al. 2007, Dutra et al. 2010), no entanto surtos de fasciolose aguda não são frequentes na região. Fasciolose crônica em bovinos jovens pode ocorrer eventualmente com sinais clínicos de emagrecimento, diarreia e edema submandibular e as perdas econômicas ocorrem, majoritariamente, devido a doença subclínica (Lotfollahzadeh et al. 2008). Um surto de fasciolose aguda em vacas com cria ao pé criadas a campo foi descrito em área endêmica na região deste estudo (Adrien et al. 2013).

Dentre todas as enfermidades diagnosticadas em ovinos observou-se que $33,6 \%$ eram parasitoses o que demonstra a grande importância dessas enfermidades para essa espécie animal. Em um estudo de doenças de ovinos na região Central do Rio Grande do Sul as parasitoses representaram $24,3 \%$ de todos os diagnósticos e dentre as parasitoses a hemoncose representou 62,5\% (Rissi et al. 2010).

As parasitoses mistas e a hemoncose representaram juntas $78,1 \%$ dos surtos de parasitoses observados em ovinos, sendo esta última, sem dúvida, uma das mais importantes parasitoses nesta espécie, devido a sua patogenicidade para o hospedeiro. Cabe ressaltar que a hemoncose afetou animais de todas as idades com média de 33,8 meses sendo, portanto, uma enfermidade de todas as categorias. Além disso, Haemonchus contortus é um dos parasitos com maior índice de resistência a anti-helmínticos determinando um impacto econômico negativo considerável na produção de ovinos na região. Na atualidade a resistência é um dos maiores problemas para o controle das parasitoses em ovinos e o teste de resistência dos parasitas aos anti-helmínticos deve ser realizado sistematicamente pelos veterinários para estabelecer planos de controle adequados (Molento 2004, Veríssimo et al. 2012).

A fasciolose ovina representou $4,4 \%$ das parasitoses, sendo que oito de 12 surtos eram provenientes de municí- pios onde a doença é endêmica. 0 número inexpressivo de surtos no período do estudo sugere que esta enfermidade é bastante conhecida nestes municípios e está no esquema de tratamento antiparasitário de rotina nas propriedades com assistência veterinária. Por esta razão, os surtos são infrequentes embora a condenação de fígados por fasciolose crônica seja elevada nos frigoríficos da região (Fiss et al. 2012).

A área de influência do LRD/UFPel do Sul (RS) possui um rebanho bovino de 2.800 .000 cabeças (IBGE 2012), a estimativa anual de mortalidade é de $5 \%$ ao ano (Riet-Correa \& Medeiros 2001), representando 140.000 bovinos mortos anualmente. 0 presente estudo demonstrou que, em média, 10,1\% de todos os diagnósticos realizados em bovinos foram devidos a doenças parasitárias, com isso, estima-se que 14.140 bovinos morrem por doenças parasitárias anualmente na região. Considerando-se um preço médio de $\mathrm{R} \$ 1.200$ por animal, calcula-se um prejuízo aproximado de $\mathrm{R} \$ 16.968 .000$ /ano.

Por sua vez, o rebanho ovino da região é de aproximadamente 1.000.000 de cabeças (IBGE 2012). A taxa de mortalidade anual para essa espécie é, também, de 5\% (SEAPA 2010), isso significa uma mortalidade anual de 50.000 ovinos. Utilizando o percentual de surtos por doenças parasitárias obtido nesse estudo (33,6\%), para esta espécie, pode-se considerar que anualmente morrem 16.800 ovinos por doenças parasitárias. Estimando-se um valor de $R \$ 120,00$ por animal, a perda econômica por mortes, nessa espécie é de aproximadamente $\mathrm{R} \$ 2.016 .000 /$ ano. Deve considerar-se que neste cálculo não estão incluídas as perdas indiretas como, por exemplo, a queda na produção (carne, leite e lã), gastos com medicamentos e assistência veterinária entre outras. Os resultados deste trabalho refletem a realidade de mais de três décadas de diagnósticos das doenças que ocorrem em animais de produção realizados na região sul do Rio Grande do Sul. A determinação da frequência e da epidemiologia das diferentes parasitoses pode ser um fator de diminuição de perdas econômicas.

Foi possível concluir que, em bovinos, na região sul do Rio Grande do Sul, os agentes da TPB são os principais causadores de perdas econômicas, seguidos pelas parasitoses mistas. Em ovinos a parasitose gastrintestinal mista e a hemoncose, juntas, representam mais de $70 \%$ dos diagnósticos realizados. As perdas por mortes em ambas as espécies devido às doenças parasitárias podem chegar a valores superiores a $\mathrm{R} \$ 18.984 .000 /$ ano. 


\section{REFERÊNCIAS}

Adrien M.L., Schild A.L., Marcolongo-Pereira C., Fiss L., Ruas J., Grecco F. \& Raffi M. 2013. Acute fasciolosis in cattle in southern Brazil. Pesq. Vet. Bras. 33(6):705-709.

Almeida M.B., Tortelli F.P., Riet-Correa B., Ferreira J.L.M., Soares M.P., Farias N.A.R., Riet-Correa F. \& Schild A.L. 2006. Tristeza parasitária bovina na região sul do Rio Grande do Sul: estudo retrospectivo de 1978-2005. Pesq. Vet. Bras. 26(4):237-242.

Amarante A.F.T. 2004. Controle integrado de helmintos de bovinos e ovinos. XIII Congresso Brasileiro de Parasitologia Veterinária e I Simpósio Latino-Americano de Ricketsioses, Ouro Preto, MG.

Borges F.A., Almeida G.D., Heckler R.P., Lemes R.T., Onizuka M.K.V. \& Borges D.G.L. 2013. Anthelmintic resistance impact on tropical beef cattle productivity: effect on weight gain of weaned calves. Trop. Anim. Health Prod. 45: 723-727.

Costa V.M.M., Simões S.V.D. \& Riet-Correa F. 2009. Doenças parasitárias em ruminantes no semi-árido brasileiro. Pesq. Vet. Bras. 9(7):563-568.

Cunha F.O.V., Marques S.M.T. \& Mattos M.J.T. 2007. Prevalência de Fasciola hepatica em ovinos no Rio Grande do Sul, Brasil. Parasitology Latinoamerican 62:188-191.

Dutra L.H., Molento M.B., Naumann C.R.C., Biondo A.W., Fortes F.S., Savio D. \& Malone J.B. 2010. Mapping risk of fasciolosis in the south of Brazil using geographic information system. Vet. Parasitol. 169:76-81.

Fiel C., Guzmán M., Steffan P., Prieto O. \& Bhushan C. 2011. Comparative efficacy of trichlorphon and trichlorphon/ivermectin combination treatment against anthelmintic-resistant cattle nematodes in Argentina. Parasitol. Res. 109(1):105-112.

Fiss L., Adrien M.L., Marcolongo-Pereira C., Assis-Brasil N.D., Ruas J.L., Sallis E.S.V., Riet-Correa F. \& Schild A.L. 2012. Subacute and acute fasciolosis in sheep in Southern Brasil. Parasitol. Res. 112(2):883-887.

IBGE 2012. Produção da Pecuária Municipal. Instituto Brasileiro de Geografia e Estatística. Disponível em <http://www.ibge.gov.br/home/ estatistica/economia/ppm/2012/ default_pdf.shtm> Acesso em 8 mai. 2015.

Lotfollahzadeh S., Mohri M., Bahadori S.R., Dezfouly M.R. \& Tajik P. 2008. The relationship between normocytic, hypochromic anaemia and iron concentration together with hepatic enzyme activities in cattle infected with Fasciola hepatica. J. Helminthol. 82(1):85-88.
Molento M.B. 2004. Resistência de helmintos em ovinos e caprinos. XIII Congresso Brasileiro de Parasitologia Veterinária e I Simpósio Latino-Americano de Ricketsioses, Ouro Preto, MG.

Molento M.B. 2005. Resistência parasitária em helmintos de equídeos e propostas de manejo. Ciência Rural 35(6):1469-1477.

Nari A. \& Rizzo E. 2004. Epidemiología y control de nematodes gastrointestinales, p.155-201. In: Nari A. \& Fiel C. (Eds), Enfermedades Parasitarias de Importancia Económica en Bovinos: bases epidemiológicas para su prevención y control en Argentina y Uruguay. Editorial Hemisferio Sur, Montevideo. 519p.

Perry B.D. \& Randolph T.F. 1999. Improving the assessment of the economic impact of parasitic diseases and of their control in production animals. Vet. Parasitol. 84:145-168.

Riet-Correa F. \& Medeiros R.M.T. 2001. Intoxicações por plantas em ruminantes no Brasil e no Uruguay: importância econômica, controle e risco para saúde pública. Pesq. Vet. Bras. 21(1):38-42.

Rissi D.R., Pierezan F., Oliveira Filho J.C., Fighera R.A., Irigoyen L.F., Kommers G.D. \& Barros C.S.L. 2010. Doenças de ovinos da região Central do Rio Grande do Sul: 361 casos. Pesq. Vet. Bras. 30(1):21-28.

Ruas J.L. 2007. Pneumonia verminótica, p.579-584. In: Riet-Correa F., Schild A.L., Lemos R.A.A. \& Borges J.R.J. (Eds), Doenças de Ruminantes e Equídeos. Vol.1. Equali, Campo Grande, MS. 722p.

Ruas J.L. \& Berne M.E.A. 2007. Parasitoses por nematódeos gastrintestinais em bovinos e ovinos, p.584-604. In: Riet-Correa F., Schild A.L., Lemos R.A.A. \& Borges J.R.J. (Eds), Doenças de Ruminantes e Equídeos. Vol.1. Equali, Campo Grande, MS. 722p.

Schild A.L., Oliveira P.A., Sallis E.S.V., Raffi M.B. \& Marcolongo-Pereira C. 2015. Doenças diagnosticadas pelo Laboratório Regional de Diagnóstico no ano 2014. Boletim do Laboratório Regional de Diagnóstico 37(1):9-27.

SEAPA/RS 2010. Programa mais Ovinos no Campo. Secretaria de Agricultura, Pecuária e Agronegócio do Rio Grande do Sul, Porto Alegre. Disponível em <http://www.agricultura.rs.gov.br/conteudo/1033/?Mais_ Ovinos_no_Campo> Acesso em 13 mai. 2015.

Veríssimo C.J., Niciura S.C.M., Alberti A.L.L., Rodrigues C.F.C., Barbosa C.M.P., Chiebao D.P., Cardoso D., Silva G.S., Pereira J.R., Margatho L.F.F., Costa R.L.D., Nardon R.F., Ueno T.E.H., Curci V.C.L.M. \& Molento M.B. 2012. Multidrug and multispecies resistance in sheep flocks from São Paulo State, Brazil. Vet. Parasitol. 187:209-216. 